\author{
N.U. KHAN AND T. USMAN
}

\title{
A NEW CLASS OF LAGUERRE-BASED GENERALIZED APOSTOL POLYNOMIALS
}

\begin{abstract}
In this paper, we introduce a unified family of Laguerre-based Apostol Bernoulli, Euler and Genocchi polynomials and derive some implicit summation formulae and general symmetry identities arising from different analytical means and applying generating functions. The result extend some known summations and identities of generalized Bernoulli, Euler and Genocchi numbers and polynomials.

KEY WORDS: Laguerre-based Apostol Bernoulli polynomials, Laguerre-based Apostol Euler polynomials, Laguerre-based Apostol Genocchi polynomials, summation formulae, symmetric identities.
\end{abstract}

AMS Mathematics Subject Classification: 11B68, 33C45, 33E20.

\section{Introduction}

The two variable Laguerre polynomials $L_{n}(x, y)$ are defined by the generating function $[2]$

$$
\sum_{n=0}^{\infty} L_{n}(x, y) \frac{t^{n}}{n !}=e^{y t} C_{0}(x t)
$$

where $C_{0}(x)$ is the 0 -th order Tricomi function [28]

$$
C_{0}(x)=\sum_{r=0}^{\infty} \frac{(-1)^{r} x^{r}}{(r !)^{2}}
$$

and are represented by the series

$$
L_{n}(x, y)=\sum_{s=0}^{n} \frac{n !(-1)^{s} y^{n-s} x^{s}}{(n-s) !(s !)^{2}} .
$$


Recently, Ozarslan [22] introduced the following unification of the Apostol Bernoulli, Apostol Euler and Apostol Genocchi polynomials. Explicitly Ozarslan studied a generating function of the form

$$
\begin{gathered}
\left(\frac{2^{1-k} t^{k}}{\beta^{b} e^{t}-a^{b}}\right)^{\alpha} e^{x t}=\sum_{n=0}^{\infty} P_{n, \beta}^{(\alpha)}(x ; k, a, b) \frac{t^{n}}{n !}, \\
\left(t+b \ln \left(\frac{\beta}{\alpha}\right)<2 \pi, k \in N_{0} ; a, b \in \Re^{+} ; \alpha \in \Re, \beta \in C\right) .
\end{gathered}
$$

We notice that for $\alpha=1$,

$$
P_{n, \beta}^{(1)}(x ; k, a, b)=P_{n, \beta}(x ; k, a, b)
$$

and then (4) reduces to

$$
\left(\frac{2^{1-k} t^{k}}{\beta^{b} e^{t}-a^{b}}\right) e^{x t}=\sum_{n=0}^{\infty} P_{n, \beta}(x ; k, a, b) \frac{t^{n}}{n !}
$$

which is defined by Ozden [23]. Ozden et al. [25] introduced many properties of the polynomials. We give some specific special cases as follows:

1. By substituting $a=b=k=1$ and $\beta=\lambda$ into (4), we have the Apostol-Bernoulli polynomials $P_{n, \lambda}^{(1)}(x ; 1,1,1)=B_{n}^{(\alpha)}(x ; \lambda)$, which are defined by means of the following generating function

$$
\left(\frac{t}{\lambda e^{t}-1}\right)^{\alpha} e^{x t}=\sum_{n=0}^{\infty} B_{n}^{(\alpha)}(x ; \lambda) \frac{t^{n}}{n !}, \quad(|t+\log \lambda|<2 \pi)
$$

(see for detail [3], [6], [9], [10], [14]-[19], [21], [24], [30] see also the references cited in each of these earlier works).

For $\lambda=\alpha=1$ in $(6)$, the result reduces to

$$
\left(\frac{t}{e^{t}-1}\right) e^{x t}=\sum_{n=0}^{\infty} B_{n}(x) \frac{t^{n}}{n !}, \quad(|t|<2 \pi)
$$

where $B_{n}(x)$ denotes the classical Bernoulli polynomials (see from example [3]-[40]; see also the reference cited in each of these earlier works).

2. By substituting $b=\alpha=1, k=0, a=-1$ and $\beta=\lambda$ into (4), we have the Apostol-Euler polynomials $P_{n, \lambda}^{(1)}(x ; 0,-1,1)=E_{n}^{(1)}(x ; \lambda)$, which are defined by means of the following generating function

$$
\left(\frac{2}{\lambda e^{t}+1}\right)^{\alpha} e^{x t}=\sum_{n=0}^{\infty} E_{n}^{(\alpha)}(x ; \lambda) \frac{t^{n}}{n !}, \quad(|t+\log \lambda|<\pi)
$$


(see for detail [3], [6], [9], [10], [14]-[19], [21], [24], [30] see also the references cited in each of these earlier works).

For $\lambda=\alpha=1$ in (8), the result reduces to

$$
\left(\frac{2}{e^{t}+1}\right) e^{x t}=\sum_{n=0}^{\infty} E_{n}(x) \frac{t^{n}}{n !}, \quad(|t|<\pi)
$$

where $E_{n}(x)$ denotes the classical Euler polynomials (see from example [5], $[6],[7],[9],[12],[31]-[33],[35]-[40]$; see also the reference cited in each of these earlier works).

3. By substituting $b=\alpha=1, k=1, a=-1$ and $\beta=\lambda$ into (4), we have the Apostol-Genocchi polynomials $P_{n, \lambda}^{(1)}(x ; 1,-1,1)=\frac{1}{2} G_{n}^{(1)}(x ; \lambda)$, which are defined by means of the following generating function

$$
\left(\frac{2 t}{\lambda e^{t}+1}\right)^{\alpha} e^{x t}=\sum_{n=0}^{\infty} G_{n}^{(\alpha)}(x ; \lambda) \frac{t^{n}}{n !}, \quad(|t+\log \lambda|<\pi)
$$

(see for detail [4], [6], [9], [10], [13]-[19], [21], [24], [30] see also the references cited in each of these earlier works).

For $\lambda=\alpha=1$ in (10), the result reduces to

$$
\left(\frac{2 t}{e^{t}+1}\right) e^{x t}=\sum_{n=0}^{\infty} G_{n}(x) \frac{t^{n}}{n !}, \quad(|t|<\pi)
$$

where $G_{n}(x)$ denotes the classical Genocchi polynomials (see from example $[5],[6],[7],[9],[12],[31]-[33],[35]-[40]$; see also the reference cited in each of these earlier works).

4. By substituting $x=0$ in the generating function (4), we obtain the corresponding unification of the generating function of Bernoulli, Euler and Genocchi numbers of higher order. Thus we have

$$
P_{n, \beta}^{(\alpha)}(0 ; k, a, b)=P_{n, \beta}^{(\alpha)}(k, a, b), \quad n \in N .
$$

The 2-variable Kampe de Feriet generalization of the Hermite polynomials [1] reads

$$
H_{n}(x, y)=n ! \sum_{r=0}^{\left[\frac{n}{2}\right]} \frac{y^{r} x^{n-2 r}}{r !(n-2 r) !}
$$

These polynomials are usually defined by the generating function

$$
e^{x t+y t^{2}}=\sum_{n=0}^{\infty} H_{n}(x, y) \frac{t^{n}}{n !}
$$


and reduce to the ordinary Hermite polynomials $H_{n}(x)$ when $y=-1$ and $x$ is replaced by $2 x$.

In this paper, we first gives the definitions of the Laguerre-based Apostol Bernoulli, Euler and Genocchi polynomials ${ }_{L} P_{n, \beta}^{(\alpha)}(x, y, z ; k, a, b)$ which generalizes the concept stated above and then investigate their basic properties and relationship with Bernoulli number $B_{n}(k, a, b)$, Bernoulli polynomials $B_{n}(x ; k, a, b)$, Euler number $E_{n}(k, a, b)$, Euler polynomials $E_{n}(x ; k, a, b)$, Genocchi number $G_{n}(k ; a, b)$ and the Genocchi polynomials $G_{n}(x ; k, a, b)$. Some implicit summation formulae and general symmetry identities of ${ }_{L} P_{n, \beta}^{(\alpha)}$ $(x, y, z ; k, a, b)$ are derived by using different analytical means and applying generating functions. These result extends some known summations and identities of generalized Hermite-Bernoulli polynomials studied by Dattoli et al, Natalini et al, Yang, Ozarslan and Pathan et al.

\section{Laguerre-based generalized Apostol-Bernoulli, Euler and Genocchi polynomials}

In this section, we will give the definition and properties of the Laguerrebased Apostol polynomials as follows:

Definition 1. Let $a, b>0$ and $a \neq b$. The generalized Laguerre-based Apostol polynomials ${ }_{L} P_{n, \beta}^{(\alpha)}(x, y, z ; k, a, b)$ for nonnegative integer $n$ are defined by

$$
\begin{aligned}
\left(\frac{2^{1-k} t^{k}}{\beta^{b} e^{t}-a^{b}}\right)^{\alpha} e^{y t+z t^{2}} C_{0}(x t)= & \sum_{n=0}^{\infty}{ }_{L} P_{n, \beta}^{(\alpha)}(x, y, z ; k, a, b) \frac{t^{n}}{n !} \\
& \left(k \in N_{0} ; a, b \in \Re /\{0\} ; \alpha, \beta \in C\right) .
\end{aligned}
$$

For the existence of the expansion, we need

(i) $|t|<2 \pi$ when $\alpha \in N_{0}, k=1$ and $\left(\frac{\beta}{a}\right)^{b}=1 ;|t|<2 \pi$ when $\alpha \in N_{0}$, $k=1,2,3, \cdots$ and $\left(\frac{\beta}{a}\right)^{b}=1 ;|t|<\left|b \log \frac{\beta}{a}\right|$ when $\alpha \in N_{0}, k \in N$ and $\left(\frac{\beta}{a}\right)^{b} \neq 1($ or $\neq 1) ; x, y, z \in \Re, \beta \in C, a, b \in C /\{0\} ; 1^{\alpha}:=1$;

(ii) $|t|<\pi$ when $\left(\frac{\beta}{a}\right)^{b}=-1 ;|t|<\left|b \log \left(\frac{\beta}{a}\right)\right|$ when $\left(\frac{\beta}{a}\right)^{b} \neq 1 ; x, y, z \in \Re$, $k=0, \alpha, \beta \in C, a, b \in C /\{0\} ; 1^{\alpha}:=1$;

(iii) $|t|<\pi$ when $\alpha \in N_{0}$ and $\left(\frac{\beta}{a}\right)^{b}=-1 ; x, y, z \in \Re, k \in N, \beta \in C$; $a, b \in C\{0\} ; 1^{\alpha}=1$, where $w=|w| e^{i \theta},-\pi \leq \theta<\pi$ and $\log (w)=$ $\log (|w|)+i \theta$.

For $k=a=b=1$ and $\beta=\lambda$ in (14), we define the following. 
Definition 2. Let $\alpha \in N_{0}, \lambda$ be an arbitrary (real or complex) parameter and $x, y, z \in \Re$, The Laguerre-based generalized Apostol-Bernoulli polynomials are defined by

$$
\left(\frac{t}{\lambda e^{t}-1}\right)^{\alpha} e^{y t+z t^{2}} C_{0}(x t)=\sum_{n=0}^{\infty}{ }_{L} B_{n}^{(\alpha)}(x, y, z ; \lambda) \frac{t^{n}}{n !}
$$

$\left(|t|<2 \pi\right.$ when $\alpha \in C$ and $\lambda=1 ;|t|<|\log (\lambda)|$ when $\alpha \in N_{0}$ and $\lambda \neq 1$; $\left.x, y, z \in \Re ; 1^{\alpha}=1\right)$. It is clear that

$$
{ }_{L} P_{n, \lambda}^{(\alpha)}(x, y, z ; 1,1,1)={ }_{L} B_{n}^{(\alpha)}(x, y, z ; \lambda) .
$$

For $k+1=-a=b=1$ and $\beta=\lambda$ in (14), we define the following.

Definition 3. Let $\alpha$ and $\lambda(\neq 1)$ be an arbitrary (real or complex) parameter and $x, y, z \in \Re$, The Laguerre-based generalized Apostol-Euler polynomials are defined by

$$
\left(\frac{2}{\lambda e^{t}+1}\right)^{\alpha} e^{y t+z t^{2}} C_{0}(x t)=\sum_{n=0}^{\infty}{ }_{L} E_{n}^{(\alpha)}(x, y, z ; \lambda) \frac{t^{n}}{n !}
$$

$(|t|<\pi$ when $\lambda=1 ;|t|<|\log (-\lambda)|$ when $\lambda \neq 1 ; x, y, z \in \Re, \alpha \in C$; $\left.1^{\alpha}=1\right)$. It is clear that

$$
{ }_{L} P_{n, \lambda}^{(\alpha)}(x, y, z ; 0,-1,1)={ }_{L} E_{n}^{(\alpha)}(x, y, z ; \lambda)
$$

For $k=-2 a=b=1$ and $2 \beta=\lambda$ in (14), we define the following.

Definition 4. Let $\alpha$ and $\lambda(\neq 1)$ be an arbitrary (real or complex) parameter and $x, y, z \in \Re$, The Laguerre-based generalized Apostol-Genocchi polynomials are defined by

$$
\left(\frac{2 t}{\lambda e^{t}+1}\right)^{\alpha} e^{y t+z t^{2}} C_{0}(x t)=\sum_{n=0}^{\infty}{ }_{L} G_{n}^{(\alpha)}(x, y, z ; \lambda) \frac{t^{n}}{n !}
$$

$\left(|t|<\pi\right.$ when $\alpha \in N_{0}$ and $\lambda=1 ;|t|<|\log (-\lambda)|$ when $\alpha \in N_{0}, \lambda \neq 1$; $\left.x, y, z \in \Re ; 1^{\alpha}=1\right)$. It is clear that

$$
{ }_{L} P_{n, \frac{\lambda}{2}}^{(\alpha)}\left(x, y, z ; 1,-\frac{1}{2}, 1\right)={ }_{L} G_{n}^{(\alpha)}(x, y, z ; \lambda) .
$$

The generalized Laguerre-based Apostol polynomials ${ }_{L} P_{n, \beta}^{(\alpha)}(x, y, z ; k, a, b)$ defined by (14) have the following properties which are stated as theorem below. 
Theorem 1. Let $a, b>0$ and $a \neq b$. Then $x, y, z \in \Re$ and $n \geq 0$. Then the following relations holds true.

$$
\begin{aligned}
& { }_{L} P_{n, \beta}^{(\alpha)}(x, y, z ; k, 1,1)={ }_{L} P_{n, \beta}^{(\alpha)}(x, y, z ; k), \\
& { }_{L} P_{n, \beta}^{(\alpha)}(x, y, z ; k, 1,1)={ }_{L} B_{n}^{(\alpha)}(x, y, z ; \lambda)
\end{aligned}
$$

$$
\begin{aligned}
& { }_{L} P_{n, \beta}^{(\alpha)}(0,0,0 ; k, a, b)=P_{n, \beta}^{(\alpha)}(k, a, b),{ }_{L} P_{n, \beta}^{(\alpha)}(x, 0,0 ; k, 1,1)=B_{n}^{(\alpha)}(x ; \lambda) \\
& { }_{L} P_{n, \beta}^{(\alpha+\gamma)}(x, y+z, v+u ; k, a, b) \\
& \quad=\sum_{k=0}^{n}\left(\begin{array}{c}
n \\
m
\end{array}\right){ }_{L} P_{n-m, \beta}^{(\alpha)}(x, z, v ; a, b, e ; \lambda)_{H} P_{m, \beta}^{(\gamma)}(y, u ; a, b, e ; \lambda)
\end{aligned}
$$

$$
\begin{aligned}
{ }_{L} P_{n, \beta}^{(\alpha+\gamma)}(x, y+v, z ; k, a, b) & \\
& =\sum_{k=0}^{n}\left(\begin{array}{c}
n \\
m
\end{array}\right){ }_{L} P_{n-m, \beta}^{(\alpha)}(x, y, z ; k, a, b) P_{m, \beta}^{(\gamma)}(v ; a, b, e ; \lambda)
\end{aligned}
$$

where ${ }_{H} P_{m, \beta}^{(\alpha)}(y, u ; a, b, e ; \lambda)$ is the generalized Hermite-based Apostol polynomials defined by means of the generating function as follows:

$$
\left(\frac{2^{1-k} t^{k}}{\beta^{b} e^{t}-a^{b}}\right)^{\alpha} e^{y t+u t^{2}}=\sum_{n=0}^{\infty}{ }_{H} P_{n, \beta}^{(\alpha)}(y, u ; k, a, b) \frac{t^{n}}{n !} .
$$

Proof. The formula in (18) are obvious. Applying definition (14), we have

$$
\begin{aligned}
\sum_{n=0}^{\infty}{ }_{L} & P_{n, \beta}^{(\alpha+\gamma)}(x, y+z, v+u ; k, a, b) \frac{t^{n}}{n !} \\
& =\left(\sum_{n=0}^{\infty}{ }_{L} P_{n, \beta}^{(\alpha)}(x, z, v ; k, a, b) \frac{t^{n}}{n !}\right)\left(\sum_{m=0}^{\infty}{ }_{H} P_{m, \beta}^{(\gamma)}(y, u ; k, a, b) \frac{t^{m}}{m !}\right) \\
& =\sum_{n=0}^{\infty}\left(\sum_{m=0}^{n}\left(\begin{array}{c}
n \\
m
\end{array}\right){ }_{L} P_{n-m, \beta}^{(\alpha)}(x, z, v ; k, a, b)_{H} P_{m, \beta}^{(\gamma)}(y, u ; k, a, b)\right) \frac{t^{n}}{n !}
\end{aligned}
$$

Now equating the coefficients of $\frac{t^{n}}{n !}$ in the above equation, we get the result (19). Again by definition (14) of Laguerre-based Apostol polynomials, we have

$$
\begin{gathered}
\sum_{n=0}^{\infty}{ }_{L} P_{n, \beta}^{(\alpha+\gamma)}(x, y+v, z ; k, a, b) \frac{t^{n}}{n !}=\left(\frac{2^{1-k} t^{k}}{\beta^{b} e^{t}-a^{b}}\right)^{\alpha+\gamma} e^{(y+v) t+z t^{2}} C_{0}(x t) \\
=\left(\left(\frac{2^{1-k} t^{k}}{\beta^{b} e^{t}-a^{b}}\right)^{\alpha} e^{y t+z t^{2}} C_{0}(x t)\right)\left(\left(\frac{2^{1-k} t^{k}}{\beta^{b} e^{t}-a^{b}}\right)^{\gamma} e^{v t}\right)
\end{gathered}
$$


which can be written as

$$
\begin{aligned}
& =\sum_{n=0}^{\infty}{ }_{L} P_{n, \beta}^{(\alpha)}(x, y, z ; k, a, b) \frac{t^{n}}{n !} \sum_{m=0}^{\infty} P_{m, \beta}^{(\gamma)}(v ; k, a, b) \frac{t^{m}}{m !} \\
& =\sum_{n=0}^{\infty}\left(\sum_{m=0}^{n}\left(\begin{array}{c}
n \\
m
\end{array}\right){ }_{L} P_{n-m, \beta}^{(\alpha)}(x, y, z ; k, a, b) P_{m}^{(\gamma)}(v ; k, a, b)\right) \frac{t^{n}}{n !}
\end{aligned}
$$

On equating the coefficient of the like power of $\frac{t^{n}}{n !}$ in the above equation, we get the result (20). Hence we complete the proof of theorem.

\section{Implicit summation formulae involving Laguerre- based Apostol polynomials}

This section of the paper is devoted to employing the definition of the Laguerre-based Apostol polynomials ${ }_{L} P_{n, \beta}^{(\alpha)}(x, y, z ; k, a, b)$ to obtain finite summations. For the derivation of implicit formulae involving the Laguerrebased Apostol polynomials ${ }_{L} P_{n, \beta}^{(\alpha)}(x, y, z ; k, a, b)$ the same consideration as developed for the ordinary Hermite and related polynomials in Khan et al. [8] and Hermite-based polynomials in Ozarslan [21] holds as well. First we prove the following results involving Laguerre-based Apostol polynomials ${ }_{L} P_{n, \beta}^{(\alpha)}(x, y, z ; k, a, b)$.

Theorem 2. Let $a, b>0$ and $a \neq b$. Then for $x, y, z \in \Re$ and $n \geq 0$. The following implicit summation formula for Laguerre-based Apostol polynomials ${ }_{L} P_{n, \beta}^{(\alpha)}(x, y, z ; k, a, b)$ holds true:

$$
\begin{aligned}
& { }_{L} P_{m+n}^{(\alpha)}(x, v, z ; k, a, b) \\
& \quad=\sum_{p, q=0}^{m, n}\left(\begin{array}{c}
n \\
p
\end{array}\right)\left(\begin{array}{c}
m \\
q
\end{array}\right)(v-y)^{p+q}{ }_{L} P_{m+n-p-q}^{(\alpha)}(x, y, z ; k, a, b) .
\end{aligned}
$$

Proof. We replace $t$ by $t+u$ and rewrite the generating function (14) as

$$
\begin{aligned}
& \left(\frac{2^{1-k}(t+u)^{k}}{\beta^{b} e^{(t+u)}-a^{b}}\right)^{\alpha} e^{z(t+u)^{2}} C_{0}(x(t+u)) \\
& \quad=e^{-y(t+u)} \sum_{m, n=0}^{\infty} L_{m+n, \beta}^{(\alpha)}(x, y, z ; k, a, b) \frac{t^{n}}{n !} \frac{u^{m}}{m !}
\end{aligned}
$$


Replacing $y$ by $v$ in the above equation and equating the resulting equation to the above equation, we get

$$
\begin{array}{r}
e^{(v-y)(t+u)} \sum_{m, n=0}^{\infty}{ }_{L} P_{m+n, \beta}^{(\alpha)}(x, y, z ; k, a, b) \frac{t^{n}}{n !} \frac{u^{m}}{m !} \\
=\sum_{m, n=0}^{\infty}{ }_{L} P_{m+n, \beta}^{(\alpha)}(x, v, z ; k, a, b) \frac{t^{n}}{n !} \frac{u^{m}}{m !} .
\end{array}
$$

On expanding exponential function (24) gives

$$
\begin{aligned}
\sum_{N=0}^{\infty} \frac{[(v-y)(t+u)]^{N}}{N !} & \sum_{m, n=0}^{\infty}{ }_{L} P_{m+n, \beta}^{(\alpha)}(x, y, z ; k, a, b) \frac{t^{n}}{n !} \frac{u^{m}}{m !} \\
& =\sum_{m, n=0}^{\infty}{ }_{L} P_{m+n, \beta}^{(\alpha)}(x, v, z ; k, a, b) \frac{t^{n}}{n !} \frac{u^{m}}{m !}
\end{aligned}
$$

which on using formula $[34$, p. $52(2)]$

$$
\sum_{N=0}^{\infty} f(N) \frac{(x+y)^{N}}{N !}=\sum_{n, m=0}^{\infty} f(m+n) \frac{x^{n}}{n !} \frac{y^{m}}{m !}
$$

in the left hand side becomes

$$
\begin{aligned}
\sum_{p, q=0}^{\infty} \frac{(v-y)^{p+q} t^{p} u^{q}}{p ! q !} & \sum_{m, n=0}^{\infty}{ }_{L} P_{m+n, \beta}^{(\alpha)}(x, y, z ; k, a, b) \frac{t^{n}}{n !} \frac{u^{m}}{m !} \\
& =\sum_{m, n=0}^{\infty}{ }_{L} P_{m+n, \beta}^{(\alpha)}(x, v, z ; k, a, b) \frac{t^{n}}{n !} \frac{u^{m}}{m !} .
\end{aligned}
$$

Now replacing $n$ by $n-p, m$ by $m-q$ and using the lemma [34, p. 100(1)] in the left hand side of $(27)$, we get

$$
\begin{aligned}
\sum_{p, q=0}^{\infty} & \sum_{m, n=0}^{\infty} \frac{(v-y)^{p+q}}{p ! q !}{ }_{L} P_{m+n-p-q, \beta}^{(\alpha)}(x, y, z ; k, a, b) \frac{t^{n}}{(n-p) !} \frac{u^{m}}{(m-q) !} \\
= & \sum_{m, n=0}^{\infty}{ }_{L} P_{m+n, \beta}^{(\alpha)}(x, v, z ; k, a, b) \frac{t^{n}}{n !} \frac{u^{m}}{m !}
\end{aligned}
$$

Finally, on equating the coefficients of the like powers of $t$ and $u$ in the above equation, we get the required result. 
Corollary 1. Let $a, b>0, a \neq b$ and $k=1$. Then for $x, y, z \in \Re$ and $n \geq 0$. The following implicit summation formula for Laguerre-based generalized Apostol-Bernoulli polynomials ${ }_{L} B_{n}^{(\alpha)}(x, y, z ; \lambda, a, b)$ holds true:

$$
\begin{aligned}
& { }_{L} B_{m+n}^{(\alpha)}(x, v, z ; \lambda, a, b) \\
& \quad=\sum_{p, q=0}^{m, n}\left(\begin{array}{c}
n \\
p
\end{array}\right)\left(\begin{array}{c}
m \\
q
\end{array}\right)(v-y)^{p+q} B_{m+n-p-q}^{(\alpha)}(x, y, z ; \lambda, a, b) .
\end{aligned}
$$

Corollary 2. Let $a, b>0, a \neq b$ and $k=0$. Then for $x, y, z \in \Re$ and $n \geq 0$. The following implicit summation formula for Laguerre-based generalized Apostol-Euler polynomials ${ }_{L} E_{n}^{(\alpha)}(x, y, z ; \lambda, a, b)$ holds true:

$$
\begin{aligned}
& { }_{L} E_{m+n}^{(\alpha)}(x, v, z ; \lambda, a, b) \\
& \quad=\sum_{p, q=0}^{m, n}\left(\begin{array}{c}
n \\
p
\end{array}\right)\left(\begin{array}{c}
m \\
q
\end{array}\right)(v-y)^{p+q}{ }_{L} E_{m+n-p-q}^{(\alpha)}(x, y, z ; \lambda, a, b) .
\end{aligned}
$$

Corollary 3. Let $a, b>0, a \neq b$ and $k=1$. Then for $x, y, z \in \Re$ and $n \geq 0$. The following implicit summation formula for Laguerre-based generalized Apostol-Genocchi polynomials ${ }_{L} G_{n}^{(\alpha)}(x, y, z ; \lambda, a, b)$ holds true:

$$
\begin{aligned}
& { }_{L} G_{m+n}^{(\alpha)}(x, v, z ; \lambda, a, b) \\
& \quad=\sum_{p, q=0}^{m, n}\left(\begin{array}{c}
n \\
p
\end{array}\right)\left(\begin{array}{c}
m \\
q
\end{array}\right)(v-y)^{p+q}{ }_{L} G_{m+n-p-q}^{(\alpha)}(x, y, z ; \lambda, a, b) .
\end{aligned}
$$

Theorem 3. Let $a, b>0$ and $a \neq b$. Then for $x, y, z \in \Re$ and $n \geq 0$. The following implicit summation formula for Laguerre-based Apostol polynomials ${ }_{L} P_{n, \beta}^{(\alpha)}(x, y, z ; k, a, b)$ holds true:

$$
{ }_{L} P_{n, \beta}^{(\alpha)}(x, y+u, z ; k, a, b)=\sum_{j=0}^{n}\left(\begin{array}{c}
n \\
j
\end{array}\right) u^{j}{ }_{L} P_{n-j, \beta}^{(\alpha)}(x, y, z ; k, a, b) .
$$

\section{Proof. Since}

$$
\begin{gathered}
\sum_{n=0}^{\infty}{ }_{L} P_{n, \beta}^{(\alpha)}(x, y+u, z ; k, a, b) \frac{t^{n}}{n !}=\left(\frac{2^{1-k} t^{k}}{\beta^{b} e^{t}-a^{b}}\right)^{\alpha} e^{(y+u) t+z t^{2}} C_{0}(x t) \\
\sum_{n=0}^{\infty}{ }_{L} P_{n, \beta}^{(\alpha)}(x, y+u, z ; k, a, b) \frac{t^{n}}{n !}=\left(\sum_{n=0}^{\infty}{ }_{L} P_{n, \beta}^{(\alpha)}(x, y, z ; k, a, b) \frac{t^{n}}{n !}\right)\left(\sum_{j=0}^{\infty} u^{j} \frac{t^{j}}{j !}\right)
\end{gathered}
$$

Now, replacing $n$ by $n-j$ and comparing the coefficients of $t^{n}$, we get the result (32). 
Corollary 4. Let $a, b>0, a \neq b$ and $k=1$. Then for $x, y, z \in \Re$ and $n \geq 0$. The following implicit summation formula for Laguerre-based generalized Apostol-Bernoulli polynomials ${ }_{L} B_{n}^{(\alpha)}(x, y, z ; \lambda, a, b)$ holds true:

$$
{ }_{L} B_{n}^{(\alpha)}(x, y+u, z ; \lambda, a, b)=\sum_{j=0}^{n}\left(\begin{array}{c}
n \\
j
\end{array}\right) u^{j}{ }_{L} B_{n-j}^{(\alpha)}(x, y, z ; \lambda, a, b) .
$$

Corollary 5. Let $a, b>0, a \neq b$ and $k=0$. Then for $x, y, z \in \Re$ and $n \geq 0$. The following implicit summation formula for Laguerre-based generalized Apostol-Euler polynomials ${ }_{L} E_{n}^{(\alpha)}(x, y, z ; \lambda, a, b)$ holds true:

$$
{ }_{L} E_{n}^{(\alpha)}(x, y+u, z ; \lambda, a, b)=\sum_{j=0}^{n}\left(\begin{array}{c}
n \\
j
\end{array}\right) u^{j}{ }_{L} E_{n-j}^{(\alpha)}(x, y, z ; \lambda, a, b) .
$$

Corollary 6. Let $a, b>0, a \neq b$ and $k=1$. Then for $x, y, z \in \Re$ and $n \geq 0$. The following implicit summation formula for Laguerre-based generalized Apostol-Genocchi polynomials ${ }_{L} G_{n}^{(\alpha)}(x, y, z ; \lambda, a, b)$ holds true:

$$
{ }_{L} G_{n}^{(\alpha)}(x, y+u, z ; \lambda, a, b)=\sum_{j=0}^{n}\left(\begin{array}{c}
n \\
j
\end{array}\right) u^{j}{ }_{L} G_{n-j}^{(\alpha)}(x, y, z ; \lambda, a, b) .
$$

Theorem 4. Let $a, b>0$ and $a \neq b$. Then for $x, y, z \in \Re$ and $n \geq 0$. The following implicit summation formula for Laguerre-based Apostol polynomials ${ }_{L} P_{n, \beta}^{(\alpha)}(x, y, z ; k, a, b)$ holds true:

$$
\begin{aligned}
{ }_{L} P_{n, \beta}^{(\alpha)}(x, y & +u, z+w ; k, a, b) \\
= & \sum_{m=0}^{n}\left(\begin{array}{c}
n \\
m
\end{array}\right){ }_{L} P_{n-m, \beta}^{(\alpha)}(x, y, z ; k, a, b) H_{m}(u, w) .
\end{aligned}
$$

Proof. By the definition of Laguerre-based Apostol polynomials and the definition (13), we have

$$
\begin{aligned}
& \left(\frac{2^{1-k} t^{k}}{\beta^{b} e^{t}-a^{b}}\right)^{\alpha} e^{(y+u) t+(z+w) t^{2}} C_{0}(x t) \\
& =\left(\sum_{n=0}^{\infty}{ }_{L} P_{n, \beta}^{(\alpha)}(x, y, z ; k, a, b) \frac{t^{n}}{n !}\right)\left(\sum_{m=0}^{\infty} H_{m}(u, w) \frac{t^{m}}{m !}\right) .
\end{aligned}
$$

Now, replacing $n$ by $n-m$ and comparing the coefficients of $t^{n}$, we get the result (36). 
Corollary 7. Let $a, b>0, a \neq b$ and $k=1$. Then for $x, y, z \in \Re$ and $n \geq 0$. The following implicit summation formula for Laguerre-based generalized Apostol-Bernoulli polynomials ${ }_{L} B_{n}^{(\alpha)}(x, y, z ; \lambda, a, b)$ holds true:

$$
\begin{aligned}
{ }_{L} B_{n}^{(\alpha)}(x, y & +u, z+w ; \lambda, a, b) \\
= & \sum_{m=0}^{n}\left(\begin{array}{c}
n \\
m
\end{array}\right){ }_{L} B_{n-m}^{(\alpha)}(x, y, z ; \lambda, a, b) H_{m}(u, w) .
\end{aligned}
$$

Corollary 8. Let $a, b>0, a \neq b k=0$. Then for $x, y, z \in \Re$ and $n \geq 0$. The following implicit summation formula for Laguerre-based generalized Apostol-Euler polynomials ${ }_{L} E_{n}^{(\alpha)}(x, y, z ; \lambda, a, b)$ holds true:

$$
\begin{aligned}
{ }_{L} E_{n}^{(\alpha)}(x, y & +u, z+w ; \lambda, a, b) \\
= & \sum_{m=0}^{n}\left(\begin{array}{c}
n \\
m
\end{array}\right){ }_{L} E_{n-m}^{(\alpha)}(x, y, z ; \lambda, a, b) H_{m}(u, w) .
\end{aligned}
$$

Corollary 9. Let $a, b>0, a \neq b$ and $k=1$. Then for $x, y, z \in \Re$ and $n \geq 0$. The following implicit summation formula for Laguerre-based generalized Apostol-Genocchi polynomials ${ }_{L} G_{n}^{(\alpha)}(x, y, z ; \lambda, a, b)$ holds true:

$$
\begin{aligned}
{ }_{L} G_{n}^{(\alpha)}(x, y & +u, z+w ; \lambda, a, b) \\
= & \sum_{m=0}^{n}\left(\begin{array}{c}
n \\
m
\end{array}\right){ }_{L} G_{n-m}^{(\alpha)}(x, y, z ; \lambda, a, b) H_{m}(u, w) .
\end{aligned}
$$

Theorem 5. Let $a, b>0$ and $a \neq b$. Then for $x, y, z \in \Re$ and $n \geq 0$. The following implicit summation formula for Laguerre-based Apostol polynomials ${ }_{L} P_{n, \beta}^{(\alpha)}(x, y, z ; k, a, b)$ holds true:

$$
{ }_{L} P_{n, \beta}^{(\alpha)}(x, y, z ; k, a, b)=\sum_{m=0}^{n-2 j} \sum_{j=0}^{\left[\frac{n}{2}\right]} \frac{P_{m, \beta}^{(\alpha)}(k ; a, b) L_{n-m-2 j}(x, y) z^{j} n !}{m ! j !(n-m-2 j) !} .
$$

Proof. Applying the definition (14) to the term $\left(\frac{2^{1-k} t^{k}}{\beta^{b} e^{t}-a^{b}}\right)^{\alpha}$ and expanding the exponential and tricomi function $e^{y t+z t^{2}} C_{0}(x t)$ at $t=0$ yields

$$
\begin{aligned}
& \left(\frac{2^{1-k} t^{k}}{\beta^{b} e^{t}-a^{b}}\right)^{\alpha} e^{y t+z t^{2}} C_{0}(x t) \\
& =\left(\sum_{m=0}^{\infty} P_{m, \beta}^{(\alpha)}(k ; a, b) \frac{t^{m}}{m !}\right)\left(\sum_{n=0}^{\infty} L_{n}(x, y) \frac{t^{n}}{n !}\right)\left(\sum_{j=0}^{\infty} z^{j} \frac{t^{2 j}}{j !}\right)
\end{aligned}
$$




$$
\begin{aligned}
& \sum_{n=0}^{\infty}{ }_{L} P_{n, \beta}^{(\alpha)}(x, y, z ; k, a, b) \frac{t^{n}}{n !} \\
& \quad=\sum_{n=0}^{\infty}\left(\sum_{m=0}^{n} P_{m, \beta}^{(\alpha)}(k ; a, b) L_{n-m}(x, y)\right) \frac{t^{n}}{m !(n-m) !}\left(\sum_{j=0}^{\infty} z^{j} \frac{t^{2 j}}{j !}\right)
\end{aligned}
$$

Now, replacing $n$ by $n-2 j$ and comparing the coefficients of $t^{n}$, we get the result (40).

Corollary 10. Let $a, b>0, a \neq b$ and $k=1$. Then for $x, y, z \in \Re$ and $n \geq 0$. The following implicit summation formula for Laguerre-based generalized Apostol-Bernoulli polynomials ${ }_{L} B_{n}^{(\alpha)}(x, y, z ; \lambda, a, b)$ holds true:

$$
{ }_{L} B_{n}^{(\alpha)}(x, y, z ; \lambda, a, b)=\sum_{m=0}^{n-2 j} \sum_{j=0}^{\left[\frac{n}{2}\right]} \frac{B_{m}^{(\alpha)}(\lambda ; a, b) L_{n-m-2 j}(x, y) z^{j} n !}{m ! j !(n-m-2 j) !} .
$$

Corollary 11. Let $a, b>0, a \neq b$ and $k=0$. Then for $x, y, z \in \Re$ and $n \geq 0$. The following implicit summation formula for Laguerre-based generalized Apostol-Euler polynomials ${ }_{L} E_{n}^{(\alpha)}(x, y, z ; \lambda, a, b)$ holds true:

$$
{ }_{L} E_{n}^{(\alpha)}(x, y, z ; \lambda, a, b)=\sum_{m=0}^{n-2 j} \sum_{j=0}^{\left[\frac{n}{2}\right]} \frac{E_{m}^{(\alpha)}(\lambda ; a, b) L_{n-m-2 j}(x, y) z^{j} n !}{m ! j !(n-m-2 j) !}
$$

Corollary 12. Let $a, b>0, a \neq b$ and $k=1$. Then for $x, y, z \in \Re$ and $n \geq 0$. The following implicit summation formula for Laguerre-based generalized Apostol-Genocchi polynomials ${ }_{L} G_{n}^{(\alpha)}(x, y, z ; \lambda, a, b)$ holds true:

$$
{ }_{L} G_{n}^{(\alpha)}(x, y, z ; \lambda, a, b)=\sum_{m=0}^{n-2 j} \sum_{j=0}^{\left[\frac{n}{2}\right]} \frac{G_{m}^{(\alpha)}(\lambda ; a, b) L_{n-m-2 j}(x, y) z^{j} n !}{m ! j !(n-m-2 j) !} .
$$

Theorem 6. Let $a, b>0$ and $a \neq b$. Then for $x, y, z \in \Re$ and $n \geq 0$. The following implicit summation formula for Laguerre-based Apostol polynomials ${ }_{L} P_{n, \beta}^{(\alpha)}(x, y, z ; k, a, b)$ holds true:

$$
{ }_{L} P_{n, \beta}^{(\alpha)}(x, y+1, z ; k, a, b)=\sum_{j, m=0}^{n} \frac{n !(-1)^{j}(x)^{j}{ }_{H} P_{n-j-m, \beta}^{(\alpha)}(y, z ; k, a, b)}{(n-j-m) ! m !(j !)^{2}}
$$


Proof. By the definition of Laguerre-based Apostol polynomials, we have

$$
\begin{aligned}
\sum_{n=0}^{\infty}{ }_{L} & P_{n, \beta}^{(\alpha)}(x, y+1, z ; k, a, b) \frac{t^{n}}{n !}=\left(\frac{2^{1-k} t^{k}}{\beta^{b} e^{t}-a^{b}}\right)^{\alpha} e^{(y+1) t+z t^{2} C_{0}(x t)} \\
& =\left(\sum_{n=0}^{\infty}\left(\sum_{m=0}^{n} \frac{{ }_{H} P_{n-m, \beta}^{(\alpha)}(y, z ; k, a, b)}{(n-m) ! n !}\right) t^{n}\right)\left(\sum_{j=0}^{\infty} \frac{(-1)^{j}(x t)^{j}}{(j !)^{2}}\right) \\
& =\left(\sum_{n=0}^{\infty}\left(\sum_{j=0}^{\infty} \sum_{m=0}^{n} \frac{(-1)^{j}(x)^{j}{ }_{H} P_{n-m, \beta}^{(\alpha)}(y, z ; k, a, b)}{(n-m) ! n !(j !)^{2}}\right) t^{n+j}\right) .
\end{aligned}
$$

Replacing $n$ by $n-j$, we have

$$
\begin{aligned}
\sum_{n=0}^{\infty}{ }_{L} P_{n, \beta}^{(\alpha)}(x, y+1, z ; k, a, b) \frac{t^{n}}{n !} & \\
& =\left(\sum_{n=0}^{\infty}\left(\sum_{j, m=0}^{n} \frac{(-1)^{j}(x)^{j}{ }_{H} P_{n-m, \beta}^{(\alpha)}(y, z ; k, a, b)}{(n-m) ! n !(j !)^{2}}\right) t^{n+j}\right) .
\end{aligned}
$$

On comparing the coefficients of $t^{n}$, we get the result (44).

Corollary 13. Let $a, b>0, a \neq b$ and $k=1$. Then for $x, y, z \in \Re$ and $n \geq 0$. The following implicit summation formula for Laguerre-based generalized Apostol-Bernoulli polynomials ${ }_{L} B_{n}^{(\alpha)}(x, y, z ; \lambda, a, b)$ holds true:

$$
{ }_{L} B_{n}^{(\alpha)}(x, y+1, z ; \lambda, a, b)=\sum_{j, m=0}^{n} \frac{n !(-1)^{j}(x)^{j}{ }_{H} B_{n-j-m}^{(\alpha)}(y, z ; \lambda, a, b)}{(n-j-m) ! m !(j !)^{2}} .
$$

Corollary 14. Let $a, b>0, a \neq b$ and $k=0$. Then for $x, y, z \in \Re$ and $n \geq 0$. The following implicit summation formula for Laguerre-based generalized Apostol-Euler polynomials ${ }_{L} E_{n}^{(\alpha)}(x, y, z ; \lambda, a, b)$ holds true:

$$
{ }_{L} E_{n}^{(\alpha)}(x, y+1, z ; \lambda, a, b)=\sum_{j, m=0}^{n} \frac{n !(-1)^{j}(x)^{j}{ }_{H} E_{n-j-m}^{(\alpha)}(y, z ; \lambda, a, b)}{(n-j-m) ! m !(j !)^{2}} .
$$

Corollary 15. Let $a, b>0, a \neq b$ and $k=1$. Then for $x, y, z \in \Re$ and $n \geq 0$. The following implicit summation formula for Laguerre-based generalized Apostol-Genocchi polynomials ${ }_{L} G_{n}^{(\alpha)}(x, y, z ; \lambda, a, b)$ holds true:

$$
{ }_{L} G_{n}^{(\alpha)}(x, y+1, z ; \lambda, a, b)=\sum_{j, m=0}^{n} \frac{n !(-1)^{j}(x)^{j}{ }_{H} G_{n-j-m}^{(\alpha)}(y, z ; \lambda, a, b)}{(n-j-m) ! m !(j !)^{2}}
$$


Theorem 7. Let $a, b>0$ and $a \neq b$. Then for $x, y, z \in \Re$ and $n \geq 0$. The following implicit summation formula for Laguerre-based Apostol polynomials ${ }_{L} P_{n, \beta}^{(\alpha)}(x, y, z ; k, a, b)$ holds true:

$$
{ }_{L} P_{n, \beta}^{(\alpha)}(x, y+1, z ; k, a, b)=\sum_{m=0}^{n}\left(\begin{array}{c}
n \\
m
\end{array}\right){ }_{L} P_{n-m, \beta}^{(\alpha)}(x, y, z ; k, a, b) .
$$

Proof. By the definition of Laguerre-based Apostol polynomials, we have

$$
\begin{aligned}
\sum_{n=0}^{\infty}{ }_{L} & P_{n, \beta}^{(\alpha)}(x, y+1, z ; k, a, b) \frac{t^{n}}{n !}-\sum_{n=0}^{\infty}{ }_{L} P_{n, \beta}^{(\alpha)}(x, y, z ; k, a, b) \frac{t^{n}}{n !} \\
= & \left(\frac{2^{1-k} t^{k}}{\beta^{b} e^{t}-a^{b}}\right)^{\alpha}\left(e^{t}-1\right) e^{y t+z t^{2}} C_{0}(x t) \\
= & \sum_{n=0}^{\infty}{ }_{L} P_{n, \beta}^{(\alpha)}(x, y, z ; k, a, b) \frac{t^{n}}{n !}\left(\sum_{m=0}^{\infty} \frac{t^{m}}{m !}-1\right) \\
= & \sum_{n=0}^{\infty}{ }_{L} P_{n}^{(\alpha)}(x, y, z ; k, a, b) \frac{t^{n}}{n !} \sum_{m=0}^{\infty} \frac{t^{m}}{m !}-\sum_{n=0}^{\infty}{ }_{L} E_{n, \beta}^{(\alpha)}(x, y, z ; k, a, b) \frac{t^{n}}{n !} \\
= & \sum_{n=0}^{\infty} \sum_{m=0}^{n}{ }_{L} P_{n-m, \beta}^{(\alpha)}(x, y, z ; k, a, b) \frac{t^{n}}{m !(n-m) !} \\
& -\sum_{n=0}^{\infty}{ }_{L} P_{n, \beta}^{(\alpha)}(x, y, z ; k, a, b) \frac{t^{n}}{n !} .
\end{aligned}
$$

Finally equating the coefficients of the like powers of $t^{n}$, we get the result (48).

Corollary 16. Let $a, b>0, a \neq b$ and $k=1$. Then for $x, y, z \in \Re$ and $n \geq 0$. The following implicit summation formula for Laguerre-based generalized Apostol-Bernoulli polynomials ${ }_{L} B_{n}^{(\alpha)}(x, y, z ; \lambda, a, b)$ holds true:

$$
{ }_{L} B_{n}^{(\alpha)}(x, y+1, z ; \lambda, a, b)=\sum_{m=0}^{n}\left(\begin{array}{c}
n \\
m
\end{array}\right){ }_{L} B_{n-m}^{(\alpha)}(x, y, z ; \lambda, a, b) .
$$

Corollary 17. Let $a, b>0, a \neq b$ and $k=0$. Then for $x, y, z \in \Re$ and $n \geq 0$. The following implicit summation formula for Laguerre-based generalized Apostol-Euler polynomials ${ }_{L} E_{n}^{(\alpha)}(x, y, z ; \lambda, a, b)$ holds true:

$$
{ }_{L} E_{n}^{(\alpha)}(x, y+1, z ; \lambda, a, b)=\sum_{m=0}^{n}\left(\begin{array}{c}
n \\
m
\end{array}\right){ }_{L} E_{n-m}^{(\alpha)}(x, y, z ; \lambda, a, b) .
$$


Corollary 18. Let $a, b>0, a \neq b$ and $k=1$. Then for $x, y, z \in \Re$ and $n \geq 0$. The following implicit summation formula for Laguerre-based generalized Apostol-Genocchi polynomials ${ }_{L} G_{n}^{(\alpha)}(x, y, z ; \lambda, a, b)$ holds true:

$$
{ }_{L} G_{n}^{(\alpha)}(x, y+1, z ; \lambda, a, b)=\sum_{m=0}^{n}\left(\begin{array}{c}
n \\
m
\end{array}\right){ }_{L} G_{n-m}^{(\alpha)}(x, y, z ; \lambda, a, b) .
$$

\section{General symmetry identities for Laguerre-based Apostol polynomials}

In this section, we give general symmetry identities for the Laguerre-based Apostol polynomials ${ }_{L} P_{n, \beta}^{(\alpha)}(x, y, z ; k, a, b)$ by applying the generating function (14). The result extends some known identities of Zhang et al. [42], Yang et al. [41], Pathan [26], Pathan and Khan [27] and Ozarslan [21]. Throughout this section $\alpha$, will be considered as an arbitrary real or a complex parameter.

Theorem 8. Let $\alpha, k \in N_{0} ; a, b \in \Re /\{0\} ; \beta \in C, x, y, z \in \Re$ and $n \geq 0$. Then the following identity holds true:

$$
\begin{gathered}
\sum_{m=0}^{n}\left(\begin{array}{c}
n \\
m
\end{array}\right) d^{m} c^{n-m}{ }_{L} P_{n-m, \beta}^{(\alpha)}\left(x, d y, d^{2} z ; k, a, b\right)_{L} P_{m, \beta}^{(\alpha)}\left(x, c y, c^{2} z ; k, a, b\right) \\
=\sum_{m=0}^{n}\left(\begin{array}{c}
n \\
m
\end{array}\right) c^{m} d^{n-m}{ }_{L} P_{n-m, \beta}^{(\alpha)}\left(x, c y, c^{2} z ; k, a, b\right) \\
\times{ }_{L} P_{m, \beta}^{(\alpha)}\left(x, d y, d^{2} z ; k, a, b\right) .
\end{gathered}
$$

Proof. Start with

$$
g(t)=\left(\frac{c^{k} d^{k} 2^{2(1-k)} t^{2 k}}{\left(\beta^{b} e^{c t}-a^{b}\right)\left(\beta^{b} e^{d t}-a^{b}\right)}\right)^{\alpha} e^{(d+c) y t+\left(d^{2}+c^{2}\right) z t^{2}}\left[C_{0}(x t)\right]^{2}
$$

and

$$
C_{0}(c d x t) \neq C_{0}(c x t) C_{0}(d x t) .
$$

Then the expression for $g(t)$ is symmetric in $a$ and $b$ and we can expand $g(t)$ into series in two ways to obtain

$$
\begin{gathered}
g(t)=\sum_{n=0}^{\infty}{ }_{L} P_{n, \beta}^{(\alpha)}\left(x, d y, d^{2} z ; k, a, b\right) \frac{(c t)^{n}}{n !} \sum_{m=0}^{\infty}{ }_{L} P_{m, \beta}^{(\alpha)}\left(x, c y, c^{2} z ; k, a, b\right) \frac{(d t)^{m}}{m !} \\
=\sum_{n=0}^{\infty} \sum_{m=0}^{n}\left(\begin{array}{c}
n \\
m
\end{array}\right) c^{n-m} d^{m}{ }_{L} P_{n-m, \beta}^{(\alpha)}\left(x, d y, d^{2} z ; k, a, b\right) \\
\times{ }_{L} P_{m, \beta}^{(\alpha)}\left(x, c y, c^{2} z ; k, a, b\right) t^{n} .
\end{gathered}
$$


On the similar lines we can show that

$$
\begin{gathered}
g(t)=\sum_{n=0}^{\infty}{ }_{L} P_{n, \beta}^{(\alpha)}\left(x, c y, c^{2} z ; k, a, b\right) \frac{(d t)^{n}}{n !} \sum_{m=0}^{\infty}{ }_{L} P_{m, \beta}^{(\alpha)}\left(x, d y, d^{2} z ; k, a, b\right) \frac{(c t)^{m}}{m !} \\
=\sum_{n=0}^{\infty} \sum_{m=0}^{n}\left(\begin{array}{c}
n \\
m
\end{array}\right) c^{m} d^{n-m}{ }_{L} P_{n-m, \beta}^{(\alpha)}\left(x, c y, c^{2} z ; k, a, b\right) \\
\times{ }_{L} P_{m, \beta}^{(\alpha)}\left(x, d y, d^{2} z ; k, a, b\right) t^{n} .
\end{gathered}
$$

Comparing the coefficients of $t^{n}$ on the right hand sides of the last two equations we arrive at the desired result.

For $k=a=b=1$ and $\beta=\lambda$ in Theorem 8, we get the following corollary.

Theorem 9. For all $m \in N, n \in N_{0}, \lambda \in C$, we have the following symmetry identity for the Laguerre-based generalized Apostol-Bernoulli polynomials

$$
\begin{aligned}
& \sum_{m=0}^{n}\left(\begin{array}{c}
n \\
m
\end{array}\right) d^{m} c^{n-m}{ }_{L} B_{n-m}^{(\alpha)}\left(x, d y, d^{2} z ; \lambda, a, b\right)_{L} B_{m}^{(\alpha)}\left(x, c y, c^{2} z ; \lambda, a, b\right) \\
& =\sum_{m=0}^{n}\left(\begin{array}{c}
n \\
m
\end{array}\right) c^{m} d^{n-m}{ }_{L} B_{n-m}^{(\alpha)}\left(x, c y, c^{2} z ; \lambda, a, b\right)_{L} B_{m}^{(\alpha)}\left(x, d y, d^{2} z ; \lambda, a, b\right) .
\end{aligned}
$$

For $k+1=-a=b=1$ and $\beta=\lambda$ in Theorem 8, we get the following corollary.

Corollary 19. For all $m \in N, n \in N_{0}, \lambda \in C$, we have the following symmetry identity for the Laguerre-based generalized Apostol-Euler polynomials

$$
\begin{aligned}
& \sum_{m=0}^{n}\left(\begin{array}{c}
n \\
m
\end{array}\right) d^{m} c^{n-m}{ }_{L} E_{n-m}^{(\alpha)}\left(x, d y, d^{2} z ; \lambda, a, b\right)_{L} E_{m}^{(\alpha)}\left(x, c y, c^{2} z ; \lambda, a, b\right) \\
& =\sum_{m=0}^{n}\left(\begin{array}{c}
n \\
m
\end{array}\right) c^{m} d^{n-m}{ }_{L} E_{n-m}^{(\alpha)}\left(x, c y, c^{2} z ; \lambda, a, b\right)_{L} E_{m}^{(\alpha)}\left(x, d y, d^{2} z ; \lambda, a, b\right) .
\end{aligned}
$$

For $k=-2 a=b=1$ and $2 \beta=\lambda$ in Theorem 8 , we get the following corollary.

Corollary 20. For all $m \in N, n \in N_{0}, \lambda \in C$, we have the following symmetry identity for the Laguerre-based generalized Apostol-Genocchi polynomials

$$
\begin{aligned}
& \sum_{m=0}^{n}\left(\begin{array}{c}
n \\
m
\end{array}\right) d^{m} c^{n-m}{ }_{L} G_{n-m}^{(\alpha)}\left(x, d y, d^{2} z ; \lambda, a, b\right)_{L} G_{m}^{(\alpha)}\left(x, c y, c^{2} z ; \lambda, a, b\right) \\
& =\sum_{m=0}^{n}\left(\begin{array}{c}
n \\
m
\end{array}\right) c^{m} d^{n-m}{ }_{L} G_{n-m}^{(\alpha)}\left(x, c y, c^{2} z ; \lambda, a, b\right)_{L} G_{m}^{(\alpha)}\left(x, d y, d^{2} z ; \lambda, a, b\right) .
\end{aligned}
$$


Theorem 10. Let $\alpha, k \in N_{0} ; a, b \in \Re /\{0\} ; \beta \in C, x, y, z \in \Re$ and $n \geq 0$. Then the following identity holds true:

$$
\begin{gathered}
\sum_{m=0}^{n}\left(\begin{array}{c}
n \\
m
\end{array}\right) d^{m} c^{n-m} \sum_{i=0}^{c-1} \sum_{j=0}^{d-1}{ }_{L} P_{n-m, \beta}^{(\alpha)}\left(x, d y+\frac{d}{c} i+j, d^{2} u ; k, a, b\right) \\
\times{ }_{L} P_{m, \beta}^{(\alpha)}\left(x, c z, c^{2} v ; k, a, b\right) \\
=\sum_{m=0}^{n}\left(\begin{array}{c}
n \\
m
\end{array}\right) c^{m} d^{n-m} \sum_{i=0}^{d-1} \sum_{j=0}^{c-1}{ }_{L} P_{n-m, \beta}^{(\alpha)}\left(x, c y+\frac{c}{d} i+j, c^{2} u ; k, a, b\right) \\
\times{ }_{L} P_{m, \beta}^{(\alpha)}\left(x, d z, d^{2} v ; k, a, b\right) .
\end{gathered}
$$

Proof. Let

$$
\begin{aligned}
g(t)= & \left(\frac{\left(2^{2(1-k)} c^{k} d^{k} t^{2 k}\left(C_{0}(x t)\right)^{2}\right)^{\alpha}\left(e^{c d t}-1\right)^{2} e^{c d(y+z) t+c^{2} d^{2}(u+v) t^{2}}}{\left(\beta^{b} e^{c t}-a^{b}\right)^{\alpha}\left(\beta^{b} e^{d t}-a^{b}\right)^{\alpha}\left(e^{c t}-1\right)\left(e^{d t}-1\right)}\right) \\
= & \left(\frac{2^{(1-k)} c^{k} t^{k} C_{0}(x t)}{\beta^{b} e^{c t}-a^{b}}\right)^{\alpha} e^{c d y t+c^{2} d^{2} u t^{2}}\left(\frac{e^{c d t}-1}{e^{c t}-1}\right) \\
& \times\left(\frac{2^{(1-k)} d^{k} t^{k} C_{0}(x t)}{\beta^{b} e^{d t}-a^{b}}\right)^{\alpha} e^{c d z t+c^{2} d^{2} v t^{2}}\left(\frac{e^{c d t}-1}{e^{d t}-1}\right) .
\end{aligned}
$$

From where we have

$$
\begin{gathered}
=\sum_{n=0}^{\infty}\left(\sum_{m=0}^{n}\left(\begin{array}{c}
n \\
m
\end{array}\right) d^{m} c^{n-m} \sum_{i=0}^{c-1} \sum_{j=0}^{d-1}{ }_{L} P_{n-m, \beta}^{(\alpha)}\left(x, d y+\frac{d}{c} i+j, d^{2} u ; k, a, b\right)\right. \\
\left.\times{ }_{L} P_{m, \beta}^{(\alpha)}\left(x, c z, c^{2} v ; k, a, b\right)\right) \frac{t^{n}}{n !} \\
=\sum_{n=0}^{\infty}\left(\sum_{m=0}^{n}\left(\begin{array}{c}
n \\
m
\end{array}\right) c^{m} d^{n-m} \sum_{i=0}^{d-1} \sum_{j=0}^{c-1}{ }_{L} P_{n-m, \beta}^{(\alpha)}\left(x, c y+\frac{c}{d} i+j, c^{2} u ; k, a, b\right)\right. \\
\left.\times{ }_{L} P_{m, \beta}^{(\alpha)}\left(x, d z, d^{2} v ; k, a, b\right)\right) \frac{t^{n}}{n !}
\end{gathered}
$$

Our assertion follows from comparing the coefficients of $\frac{t^{n}}{n}$ on the right hand sides of the last two equations, we arrive at the desired result.

For $k=a=b=1$ and $\beta=\lambda$ in Theorem 9 , we get the following corollary. 
Corollary 21. For all $m \in N, n \in N_{0}, \lambda \in C$, we have the following symmetry identity for the Laguerre-based generalized Apostol-Bernoulli polynomials

$$
\begin{gathered}
\sum_{m=0}^{n}\left(\begin{array}{c}
n \\
m
\end{array}\right) d^{m} c^{n-m} \sum_{i=0}^{c-1} \sum_{j=0}^{d-1}{ }_{L} B_{n-m}^{(\alpha)}\left(x, d y+\frac{d}{c} i+j, d^{2} u ; \lambda, a, b\right) \\
=\sum_{m=0}^{n}\left(\begin{array}{c}
n \\
m
\end{array}\right) c^{m} d^{n-m} \sum_{i=0}^{d-1} \sum_{j=0}^{c-1}{ }_{L}^{(\alpha)} B_{n-m}^{(\alpha)}\left(x, c z, c^{2} v ; \lambda, a, b\right) \\
\times_{L} B_{m}^{(\alpha)}\left(x, d z, d^{2} v ; \lambda, a, b\right)
\end{gathered}
$$

For $k+1=-a=b=1$ and $\beta=\lambda$ in Theorem 9, we get the following corollary.

Corollary 22. For all $m \in N, n \in N_{0}, \lambda \in C$, we have the following symmetry identity for the Laguerre-based generalized Apostol-Euler polynomials

$$
\begin{gathered}
\sum_{m=0}^{n}\left(\begin{array}{c}
n \\
m
\end{array}\right) d^{m} c^{n-m} \sum_{i=0}^{c-1} \sum_{j=0}^{d-1}{ }_{L} E_{n-m}^{(\alpha)}\left(x, d y+\frac{d}{c} i+j, d^{2} u ; \lambda, a, b\right) \\
=\sum_{m=0}^{n}\left(\begin{array}{c}
n \\
m
\end{array}\right) c^{m} d^{n-m} \sum_{i=0}^{d-1} \sum_{j=0}^{c-1} E_{m}^{(\alpha)} E_{n-m}^{(\alpha)}\left(x, c z, c^{2} v ; \lambda, a, b\right) \\
\times_{L} E_{m}^{(\alpha)}\left(x, d z, d^{2} v ; \lambda, a, b\right) .
\end{gathered}
$$

For $k=-2 a=b=1$ and $2 \beta=\lambda$ in Theorem 9, we get the following corollary.

Corollary 23. For all $m \in N, n \in N_{0}, \lambda \in C$, we have the following symmetry identity for the Laguerre-based generalized Apostol-Genocchi polynomials

$$
\begin{gathered}
\sum_{m=0}^{n}\left(\begin{array}{c}
n \\
m
\end{array}\right) d^{m} c^{n-m} \sum_{i=0}^{c-1} \sum_{j=0}^{d-1}{ }_{L} G_{n-m}^{(\alpha)}\left(x, d y+\frac{d}{c} i+j, d^{2} u ; \lambda, a, b\right) \\
=\sum_{m=0}^{n}\left(\begin{array}{c}
n \\
m
\end{array}\right) c^{m} d^{n-m} \sum_{i=0}^{d-1} \sum_{j=0}^{c-1}{ }_{L} G_{m}^{(\alpha)} G_{n-m}^{(\alpha)}\left(x, c z, c^{2} v ; \lambda, a, b\right) \\
\times_{L} G_{m}^{(\alpha)}\left(x, d z, d^{2} v ; \lambda, a, b\right) .
\end{gathered}
$$


Theorem 11. Let $\alpha, k \in N_{0}, a, b \in \Re /\{0\}, \beta \in C, x, y, z \in \Re$ and $n \geq 0$. Then the following identity holds true:

$$
\begin{gathered}
\sum_{m=0}^{n}\left(\begin{array}{c}
n \\
m
\end{array}\right) d^{m} c^{n-m} \sum_{i=0}^{c-1} \sum_{j=0}^{d-1}{ }_{L} P_{n-m, \beta}^{(\alpha)}\left(x, d y+\frac{d}{c} i+j, d^{2} u ; k, a, b\right) \\
\times{ }_{L} P_{m, \beta}^{(\alpha)}\left(x, c z+\frac{c}{d} j, c^{2} v ; k, a, b\right) \\
=\sum_{m=0}^{n}\left(\begin{array}{c}
n \\
m
\end{array}\right) c^{m} d^{n-m} \sum_{i=0}^{d-1} \sum_{j=0}^{c-1}{ }_{L} P_{n-m, \beta}^{(\alpha)}\left(x, c y+\frac{c}{d} i+j, c^{2} u ; k, a, b\right) \\
\times{ }_{L} P_{m, \beta}^{(\alpha)}\left(x, d z+\frac{d}{c} j, d^{2} v ; k, a, b\right) .
\end{gathered}
$$

Proof. The Proof is similar to Theorem 9. So, we omit the proof of the theorem.

For $k=a=b=1$ and $\beta=\lambda$ in Theorem 10, we get the following corollary.

Corollary 24. For all $m \in N, n \in N_{0}, \lambda \in C$, we have the following symmetry identity for the Laguerre-based generalized Apostol-Bernoulli polynomials

$$
\begin{array}{r}
\sum_{m=0}^{n}\left(\begin{array}{c}
n \\
m
\end{array}\right) d^{m} c^{n-m} \sum_{i=0}^{c-1} \sum_{j=0}^{d-1}{ }_{L} B_{n-m}^{(\alpha)}\left(x, d y+\frac{d}{c} i+j, d^{2} u ; \lambda, a, b\right) \\
\times{ }_{L} B_{m}^{(\alpha)}\left(x, c z+\frac{c}{d} j, c^{2} v ; \lambda, a, b\right) \\
=\sum_{m=0}^{n}\left(\begin{array}{c}
n \\
m
\end{array}\right) c^{m} d^{n-m} \sum_{i=0}^{d-1} \sum_{j=0}^{c-1}{ }_{L} B_{n-m}^{(\alpha)}\left(x, c y+\frac{c}{d} i+j, c^{2} u ; \lambda, a, b\right) \\
\times{ }_{L} B_{m}^{(\alpha)}\left(x, d z+\frac{d}{c} j, d^{2} v ; \lambda, a, b\right)
\end{array}
$$

For $k+1=-a=b=1$ and $\beta=\lambda$ in Theorem 10, we get the following corollary.

Corollary 25. For all $m \in N, n \in N_{0}, \lambda \in C$, we have the following symmetry identity for the Laguerre-based generalized Apostol-Euler polynomials

$$
\begin{aligned}
\sum_{m=0}^{n}\left(\begin{array}{c}
n \\
m
\end{array}\right) d^{m} c^{n-m} \sum_{i=0}^{c-1} \sum_{j=0}^{d-1} L_{n-m}^{(\alpha)}\left(x, d y+\frac{d}{c} i+j, d^{2} u ; \lambda, a, b\right) \\
\times{ }_{L} E_{m}^{(\alpha)}\left(x, c z+\frac{c}{d} j, c^{2} v ; \lambda, a, b\right)
\end{aligned}
$$




$$
\begin{array}{r}
=\sum_{m=0}^{n}\left(\begin{array}{c}
n \\
m
\end{array}\right) c^{m} d^{n-m} \sum_{i=0}^{d-1} \sum_{j=0}^{c-1}{ }_{L} E_{n-m}^{(\alpha)}\left(x, c y+\frac{c}{d} i+j, c^{2} u ; \lambda, a, b\right) \\
\times{ }_{L} E_{m}^{(\alpha)}\left(x, d z+\frac{d}{c} j, d^{2} v ; \lambda, a, b\right)
\end{array}
$$

For $k=-2 a=b=1$ and $2 \beta=\lambda$ in Theorem 10, we get the following corollary.

Corollary 26. For all $m \in N, n \in N_{0}, \lambda \in C$, we have the following symmetry identity for the Laguerre-based generalized Apostol-Genocchi polynomials

$$
\begin{gathered}
\sum_{m=0}^{n}\left(\begin{array}{c}
n \\
m
\end{array}\right) d^{m} c^{n-m} \sum_{i=0}^{c-1} \sum_{j=0}^{d-1}{ }_{L} G_{n-m}^{(\alpha)}\left(x, d y+\frac{d}{c} i+j, d^{2} u ; \lambda, a, b\right) \\
\times{ }_{L} G_{m}^{(\alpha)}\left(x, c z+\frac{c}{d} j, c^{2} v ; \lambda, a, b\right) \\
=\sum_{m=0}^{n}\left(\begin{array}{c}
n \\
m
\end{array}\right) c^{m} d^{n-m} \sum_{i=0}^{d-1} \sum_{j=0}^{c-1}{ }_{L} G_{n-m}^{(\alpha)}\left(x, c y+\frac{c}{d} i+j, c^{2} u ; \lambda, a, b\right) \\
\times{ }_{L} G_{m}^{(\alpha)}\left(x, d z+\frac{d}{c} j, d^{2} v ; \lambda, a, b\right)
\end{gathered}
$$

Acknowledgements. The authors are thankful to the reviewer(s) for several useful comments and suggestions towards the improvement of this paper. The authors would also like to express their thanks to the editor for encouraging comments.

\section{References}

[1] BeLl E.T., Exponential polynomials, Ann. of Math., 35(1934), 258-277.

[2] Dattoli G., Torre A., Operational methods and two variable Laguerre polynomials, Atti Acadmia di Torino, 132(1998), 1-7.

[3] Dattoli G., Lorenzutta S., Cesarano C., Finite sums and generalized forms of Bernoulli polynomials, Rendiconti di Mathematica, 19(1999), 385-391.

[4] Dere R., Simsek Y., Genocchi polynomials associated with the Umbral algebra, In Press, accepted manuscript, Appl. Math. Comput., 217(2011).

[5] Dilcher K., Asymptotic behavior of Bernoulli, Euler and generalized Bernoulli polynomials, J. Approx. Theory, 49(1987), 321-330.

[6] KIM T., Some identities for the Bernoulli, the Euler and Genocchi numbers and polynomials, Adv. Stud. Contemp. Math., 20(2010), 23-28.

[7] Kim T., Rim S.H., Simsek Y., Kim D., On the analogous of Bernoulli and Euler numbers related identities and zeta and L-functions, J. Korean. Math. Soc., 45(2008), 435-453. 
[8] Khan S., Pathan M.A., HASSAN N.A.M., Yasmin G., Implicit summation formula for Hermite and related polynomials, J. Math. Anal. Appl., $344(2008), 408-416$.

[9] LuO Q.M., The multiplication formulas for the Apostol-Bernoulli and Apostol-Euler polynomials of higher order, Integral Trans. Spec. Funct., 20(2009), 377-391.

[10] Lu, DA-Qian, Generalized Tricomi and Hermite-Tricomi functions, Appl. Math. Comput., 218(9)(2012), 5090-5098.

[11] Luo Q.-M., Apostol-Euler polynomials of higher order and Gaussian hypergeometric functions, Taiwanese J. Math., 10(4)(2006), 917-925.

[12] Luo Q.-M., SRIvastava H.M., Some generalizations of the ApostolBernoulli and Apostol-Euler polynomials, J. Math. Anal. Appl., 308(1)(2005), 290-302.

[13] Luo Q.-M., Srivastava H.M., Some generalizations of the Apostol- Genocchi polynomials and the Stirling numbers of the second kind, Appl. Math. Comput., 217(2011), 5702-5728.

[14] Luo Q.-M., Srivastava H.M., Some relationships between the ApostolBernoulli and Apostol-Euler polynomials, Comput. Math. Appl., 51(2006), 631-642.

[15] Lu D.-Q., SRIvastava H.M., Some series identities involving the generalized Apostol type and related polynomials, Comput. Math. Appl., 62(9)(2011), 3591-3602.

[16] Lu D.-Q., Luo Q.-M., Some properties of the generalized Apostol-type polynomials, Bound. Value Probl., 2013, 64(2013), 1-13.

[17] Lu D.-Q., Luo Q.-M., Some unified formulas and representations for the Apostol type polynomials, Adv. Difference Equ., 2015, 137(2015), 1-16.

[18] Lu D., Xiang-Q.C.-H., Luo Q.-M., Some results for Apostol-type polynomials associated with umbral algebra, Adv. Difference Equ., 2013, 201(2013), $1-13$.

[19] Norlund N.E., Volessungen uber Dierenzenrechung, Springer, 1924.

[20] Natalini A., Pierpaolo B., A generalization of the Bernoulli polynomials, Journal of Applied Mathematics, 3(2003), 155-163.

[21] Ozarslan M.A., Hermite-Based unified Apostol-Bernoulli, Euler and Genocchi polynomials, Advan. Diff. Eqtn., doi:10.1186/1687-1847-213-116, 2013.

[22] Ozarslan M.A., Unified Apostol-Bernoulli, Euler and Genocchi polynomials, Comput. Math. Appl., 6(2011), 2452-2462.

[23] Ozden H., Unification of generating functions of the Bernoulli, Euler and Genocchi numbers and polynomials, Numerical Anal.and Appl. Math., AIP, Conf. Proc. 1281(2010), 1125-1127.

[24] Ozden H., Generating function of the unified representation of the Bernoulli, Euler and Genocchi polynomials of higher order, to appear in Numerical Anal.and Appl. Math., AIP, Conf. Proc.(2011).

[25] Ozden H., Simsek Y., Srivastava H.M., A unified presentation of the generating functions of the generalized Bernoulli, Euler and Genocchi polynomials, Comput. Math. Appl., 60(2010), 2779-2287.

[26] Pathan M.A., A new class of generalized Hermite-Bernoulli polynomials, Georgian Mathematical Journal, 19(2012), 559-573. 
[27] Pathan M.A., Khan W.A., Some implicit summation formulas and symmetric identities for the generalized Hemite-Bernoulli polynomials, Mediterr. J. Math., DOI 10.1007/s00009-014-0423-0, Springer Basel, 2014.

[28] Rainville E.D., Special functions, The Macmillan Company, New York, 1960.

[29] Rim S.H., Kim Y.H., Lee B., Kim T., Some identities of the generalized twisted Bernoulli numbers and polynomials of higher order, J. Comput. Anal. Appl., 12(2010), 695-702.

[30] Simsek Y., Generating functions of the twisted Bernoulli numbers and polynomials associated with their interpolation functions, Adv. Stud. Contemp. Math., 16(2008), 251-278.

[31] Srivastava H.M., Some formulas for the Bernoulli and Euler polynomials at rational arguments, Math. Proc. Cambridge Philos. Soc., 129(2000), 77-84.

[32] SRivastava H.M., Some generalizations and basic (or $q$-)extensions of the Bernoulli, Euler, and Genocchi polynomials, Appl. Math. Inform. Sci., 5(2011), 390-444.

[33] Srivastava H.M., Pinter A., Remarks on some relationships between the Bernoulli and Euler polynomials, Appl. Math. Lett., 17(2004), 375-380.

[34] Srivastava H.M., Manocha H.L., A treatise on generating functions, Ellis Horwood Limited, New York, 1984.

[35] Srivastava H.M., Garg M., Choudhary S., A new generalization of the Bernoulli and related polynomials, Russian J. Math. Phys., 17(2011), 251-261.

[36] Srivastava H.M., Garg M., Choudhary S., Some new families of generalized Euler and Genocchi polynomials, Integral Transform. Spec. Funct., 15(2011), 283-305.

[37] Srivastava H.M., Garg M., Choudhary S., A new generalization of the Bernoulli and related polynomials, Russian J. Math. Phys., 15(2010), 251-261.

[38] Srivastava H.M., Kurt B., Simsek Y., Some families of Genocchi type polynomials and their interpolation functions, Integral Transform Spec. Funct., 23 (2012), 919-938; see also Corrigendum, Integral Transforms Spec. Funct. 23(2012), 939-940.

[39] Srivastava H.M., Ozarslan M.A., Kaanuglu C., Some generalized Lagrange-based Apostol-Bernoulli, Apostol-Euler and Apostol-Genocchi polynomials, Russian J. Math. Phys., 20(2013), 110-120.

[40] Temppesta P., On Appell sequence of polynomials of Bernoulli and Euler type, J. Math. Anal. Appl., 341(2008), 1295-1310.

[41] YANG S.L., An identity of symmetry for the Bernoulli polynomials, Discrete Math., 308(2008), 550-554.

[42] Zhang Z., Yang H., Several identities for the generalized Apostol Bernoulli polynomials, Computers and Mathematics with Applications, 56(2008), 29932999.

\author{
N.U. KHAN \\ Department of Applied Mathematics \\ Faculty of Engineering and Technology \\ Aligarh Muslim University \\ AligARH-202002, InDIA \\ e-mail: nukhanmath@gmail.com
}


A NeW Class of LAgUerRe-BASED ...

T. USMAN

Department of Applied Mathematics

FACUlty of EngineERING AND TeChNOLOGY

Aligarh Muslim University

AligarH-202002, IndiA

e-mail: talhausman.maths@gmail.com

Received on 09.04.2016 and, in revised form, on 23.11.2016. 